\title{
Dynamics of equilibration and collisions in ultradilute quantum droplets
}

\author{
V. Cikojević $\odot,{ }^{1,2}$ L. Vranješ Markić, ${ }^{1}$ M. Pi $\odot,{ }^{3,4}$ M. Barranco, ${ }^{3,4}$ F. Ancilotto, ${ }^{5,6}$ and J. Boronat $\odot^{2,}{ }^{*}$ \\ ${ }^{1}$ University of Split, Faculty of Science, Ruđera Boškovića 33, HR-21000 Split, Croatia \\ ${ }^{2}$ Departament de Física, Universitat Politècnica de Catalunya, Campus Nord B4-B5, E-08034 Barcelona, Spain \\ ${ }^{3}$ Departament FQA, Facultat de Física, Universitat de Barcelona, Diagonal 645, 08028 Barcelona, Spain \\ ${ }^{4}$ Institute of Nanoscience and Nanotechnology (IN2UB), Universitat de Barcelona, 08028 Barcelona, Spain \\ ${ }^{5}$ Dipartimento di Fisica e Astronomia "Galileo Galilei” and CNISM, Universita di Padova, via Marzolo 8, 35122 Padova, Italy \\ ${ }^{6}$ CNR-IOM Democritos, via Bonomea 265, I-34136 Trieste, Italy
}

(Received 16 April 2021; accepted 1 November 2021; published 29 November 2021)

\begin{abstract}
Employing time-dependent density-functional theory, we have studied dynamical equilibration and binary head-on collisions of quantum droplets taking as a case of study droplets made of a ${ }^{39} \mathrm{~K}-{ }^{39} \mathrm{~K}$ Bose mixture. The phase space of collision outcomes is extensively explored by performing fully three-dimensional calculations with effective single-component Quantum Monte Carlo-based and two-component LHY-corrected mean-field functionals. We exhaustively explored the important effect—not considered in previous studies—of the initial population ratio deviating from the optimal mean-field value $N_{2} / N_{1}=\sqrt{a_{11} / a_{22}}$. Both stationary and dynamical calculations indicate sensitivity to an initial nonoptimal concentration. When three-body losses (3BL) are present our two-component approach allows to theoretically address situations in which they mainly act on one of the components of the mixture. Our approach also allows to simultaneously explore the effect on the simulation of population imbalance and 3BL, which are coupled when they act.
\end{abstract}

DOI: 10.1103/PhysRevResearch.3.043139

\section{INTRODUCTION}

The collision of liquid drops is one of the more fundamental and complex problems addressed in fluid dynamics, with implications in basic research and applications, e.g., in microfluidics, formation of rain drops, ink-jet printing, or spraying for combustion, painting, and coating [1-4]. Liquid drop collisions were also used as a model for nucleus-nucleus reactions [5] and nanoscopic ${ }^{3} \mathrm{He}$ droplets collisions [6].

Generally speaking, on collision droplets may bounce back, coalesce into a single drop, separate after temporarily forming a partially fused system, or shatter into a cloud of small droplets. The main goal of the studies on droplet-droplet collisions is to determine how the appearance of these regimes depends on the collision parameters (droplet size, velocity, impact parameter) and intrinsic properties of the liquid (viscosity, surface tension, etc.).

With the advent of the so-called "helium drop machines", it has been possible to generate ${ }^{4} \mathrm{He}$ nanodroplets by the free expansion of a supercooled gas, as reviewed, e.g., in Ref. [7]. This has allowed to extend the study of liquid droplets to the quantum regime. Indeed, helium in its two isotopic forms, ${ }^{3} \mathrm{He}$ (a fermion) and ${ }^{4} \mathrm{He}$ (a boson), is the only element in nature which may exist at zero temperature as an extended liquid or in the form of droplets.

\footnotetext{
*Corresponding author: jordi.boronat@upc.edu

Published by the American Physical Society under the terms of the Creative Commons Attribution 4.0 International license. Further distribution of this work must maintain attribution to the author(s) and the published article's title, journal citation, and DOI.
}

At the experimental droplet temperatures, $0.37 \mathrm{~K}$ for ${ }^{4} \mathrm{He}$ and $0.15 \mathrm{~K}$ for ${ }^{3} \mathrm{He}[7],{ }^{4} \mathrm{He}$ is a superfluid and ${ }^{3} \mathrm{He}$ is a normal fluid. Studies on superfluid ${ }^{4} \mathrm{He}$ droplets collisions are very scarce, see, e.g., Refs. [8-11]. It has been recently shown that the merging of superfluid ${ }^{4} \mathrm{He}$ nanodroplets may produce quantized vortices and turbulence [11-13]

In the field of ultracold Bose gases, there are also very few studies of cold gas collisions [14-16], since the only accessible phase until recently was the gaseous, confined one. The situation has changed quite recently since a selfbound, low-density liquidlike state composed by ultracold quantum Bose-Bose mixtures, first theoretically proposed by Petrov [17], has been experimentally produced [18-21]. In these mixtures, an adequately tuned interaction can lead to a regime where the mean-field energy is comparable to the Lee, Huang, and Yang (LHY) energy. The LHY energy term is a perturbative correction to the mean-field energy, first calculated with the single-component Bose gas [22,23], and later extended to two-component Bose-Bose mixtures [24-26]. This desirable feature of stabilizing the mean-field collapse in an LHY-extended theory (MF+LHY), allowing for droplet formation, is present also in low-dimensional geometries [27-29] and accounts for the stability of dipolar droplets [30,31]. Consequently, the realm of stable quantum droplets has been extended to densities much lower than those of helium droplets [32].

As pointed out in Ref. [33], the LHY term suffers from an intrinsic inconsistency with the appearance of an imaginary term in the energy of the Bose-Bose mixture. This is at variance with a single-component Bose gas, where no such imaginary term appears and where the LHY term has proven to be valid up to relatively large densities, as it was 
confirmed by first-principle Quantum Monte Carlo (QMC) calculations [34,35]. Already in the first experimental realization of a quantum Bose-Bose liquid mixture, composed by two hyperfine states of ${ }^{39} \mathrm{~K}$, there have been deviations of observed properties from the predictions of usually employed theory based on interactions described solely in terms of $s$-wave scattering lengths [17]. These effects were properly explained instead by a QMC-based functional built to include effective-range corrections [36]. Diffusion Monte Carlo (DMC) calculations indicate that inclusion of the effective range allows to extend the universality of the theory $[36,37]$, providing improved energy functionals when both the $s$-wave scattering lengths and the effective ranges are known.

Some progress in the understanding of dynamical properties of quantum Bose-Bose droplets has been made in a recent experimental study of head-on collisions between pairs of ${ }^{39} \mathrm{~K}-{ }^{39} \mathrm{~K}$ droplets [38], providing a new avenue of research. In Ref. [38], the authors discovered a highly compressible lowdensity droplet regime, not present in the world of classical liquids, when the total atom numbers in colliding droplets are small. Thus, it is not clear whether the Weber number theory [39], describing the dynamics of classical liquid collisions, can apply to ultradilute droplets.

Depending on the velocity of each droplet, the outcome of the experiment was either merging or separating the colliding droplets [38]. A critical velocity $v_{c}$ is defined [38] as the initial velocity of each droplet above (below) which the two droplets separate (merge) on colliding. A significant discrepancy was observed between the experimental critical velocity $v_{c}$ and the theoretical analysis of the experiment carried out within the MF+LHY approach [38]. The disagreement was attributed to the lack of three-body losses (3BL), which were introduced in a next step but acting on the total density. This means that both components lose their atoms such that the density ratio is constantly kept fixed at the value $\rho_{2} / \rho_{1}=\sqrt{a_{11} / a_{22}}$, which is the mean-field stability requirement $[17,40,41]$. By doing so, the agreement between theory and experiment was found to be good.

However, on the other hand, it is unclear whether this procedure may mask interesting details which might be hidden by the fact that $3 \mathrm{BL}$ unavoidably were made to act on the total density and not on the appropriate component of the bosonic mixture. We recall that experimental measures [20] have demonstrated significant differences in intensities of 3BL of different components, which cannot be taken into account by the approach of Ref. [38]. This approach might be too restrictive for its applicability to other potentially interesting situations. In particular, it excludes the possibility that the droplets in the experiment are not fully equilibrated.

In this work we present one such framework for the description of binary collisions of ultradilute quantum droplets. It uses a QMC-based functional which takes into account the effective range of the interactions and a two-component MF+LHY functional which enables the study of nonequilibrated drops and a more realistic consideration of 3BL. Our results show the relevance of the nonoptimal concentration ratio in the outcome of drop collisions and may disclose the effect that the presence of evaporated atoms may have on the collision outcome. This halo of expelled gaseous particles is known to affect the merging in the case of viscous droplet collisions [2]. The experimental results of Ref. [38] have been taken as a case of study.

This work is organized as follows. In Sec. II we lay out the basic equations of the extended LHY mean-field theory $(\mathrm{MF}+\mathrm{LHY})$. In Sec. III we present the details of our simulations. In Sec. IV we discuss the effects of the nonoptimal initial atom number ratio and $3 \mathrm{BL}$ on the stationary drop. In Sec. V A, we systematically compare the collisions results obtained within the effective single-component MF+LHY theory with those obtained using the QMC-based functional. In Sec. V B, we report results derived within the two-component framework and we investigate the influence on the collisions of both the initial population imbalance and the $3 \mathrm{BL}$ acting only on the $\left|F, m_{F}\right\rangle=|1,0\rangle$ state. Finally, Sec. VI comprises the main conclusions of our work.

\section{MF+LHY AND QMC DENSITY FUNCTIONALS}

The LHY-extended mean-field theory (MF+LHY) is based on the following density functional per unit volume $V[17,41]$,

$$
\mathcal{E}\left[\rho_{1}, \rho_{2}\right]=E_{\mathrm{MF}} / V+E_{\mathrm{LHY}} / V,
$$

where

$$
\frac{E_{\mathrm{MF}}}{V}=\frac{2 \pi \hbar^{2} a_{11}}{m} \rho_{1}^{2}+\frac{2 \pi \hbar^{2} a_{22}}{m} \rho_{2}^{2}+\frac{4 \pi \hbar^{2} a_{12}}{m} \rho_{1} \rho_{2},
$$

and

$$
\frac{E_{\mathrm{LHY}}}{V}=\frac{256 \sqrt{\pi} \hbar^{2}}{15 m}\left(a_{11} \rho_{1}\right)^{5 / 2} f\left(1, \frac{a_{12}^{2}}{a_{11} a_{22}}, \frac{a_{22} \rho_{2}}{a_{11} \rho_{1}}\right) .
$$

We have considered equal masses $m_{1}=m_{2}=m$ and the function $f$ is defined in Ref. [17],

$$
\begin{aligned}
f(1, u, x)= & \frac{1}{4 \sqrt{2}}\left[\left(1+x+\sqrt{(1-x)^{2}+4 u x}\right)^{5 / 2}\right. \\
& \left.+\left(1+x-\sqrt{(1-x)^{2}+4 u x}\right)^{5 / 2}\right] .
\end{aligned}
$$

Here $u=a_{12}^{2} /\left(a_{11} a_{22}\right)$ and $x=a_{22} \rho_{2} /\left(a_{11} \rho_{1}\right)$. The function $f$ is complex for $a_{12}^{2}>a_{11} a_{22}$, and we use the approximation $a_{12}^{2}=a_{11} a_{22}$ to keep $f$ real. By doing so, $f(1,1, x)=(1+$ $x)^{5 / 2}$ and the LHY functional reads

$$
\frac{E_{\mathrm{LHY}}}{V}=\frac{256 \sqrt{\pi} \hbar^{2}}{15 m}\left(a_{11} \rho_{1}+a_{22} \rho_{2}\right)^{5 / 2} .
$$

The above framework is the most general version of a twocomponent equal-masses Bose-Bose energy functional, and it allows for all possible $\rho_{1}$ and $\rho_{2}$ values. This functional can be reduced to an effective one-component functional, which is the one mostly used in the study of Bose-Bose mixtures, if one uses the result that the stability of a dilute Bose-Bose mixture lies in a very narrow range of optimal partial densities $\rho_{1} / \rho_{2}=\sqrt{a_{22} / a_{11}}[40,41]$. Then, for this fixed ratio of $\rho_{1} / \rho_{2}$ the MF+LHY theory can be written in the compact form

$$
\frac{E / N}{\left|E_{0}\right| / N}=-3\left(\frac{\rho}{\rho_{0}}\right)+2\left(\frac{\rho}{\rho_{0}}\right)^{3 / 2}
$$


with $\rho$ being the total density and $E_{0} / N$ and $\rho_{0}$ the equilibrium energy per particle and equilibrium total density, respectively, given by

$$
\begin{gathered}
\rho_{0}=\frac{25 \pi}{1024} \frac{\left(a_{12} / a_{11}+\sqrt{a_{22} / a_{11}}\right)^{2}}{\left(a_{22} / a_{11}\right)^{3 / 2}\left(1+\sqrt{a_{22} / a_{11}}\right)^{4}} \frac{1}{a_{11}^{3}}, \\
\frac{E_{0}}{N}=\frac{-\hbar^{2}}{m a_{11}^{2}} \frac{25 \pi^{2}}{768} \frac{\left|a_{12} / a_{11}+\sqrt{a_{22} / a_{11}}\right|^{3}}{a_{22} / a_{11}\left(1+\sqrt{a_{22} / a_{11}}\right)^{6}}=\frac{-\hbar^{2}}{2 m \xi^{2}} .
\end{gathered}
$$

We have introduced in the last equation the healing length $\xi$, obtained by equating the kinetic energy to the energy per particle at the equilibrium density:

$$
\frac{\xi}{a_{11}}=\frac{8 \sqrt{6}}{5 \pi} \sqrt{\frac{a_{22}}{a_{11}}} \frac{\left(1+\sqrt{a_{22} / a_{11}}\right)^{3}}{\left|a_{12} / a_{11}+\sqrt{a_{22} / a_{11}}\right|^{3 / 2}} .
$$

The total atom number in the reduced unit $\tilde{N}$ introduced in Ref. [17] is given by

$$
\frac{N}{\tilde{N}}=\frac{3 \sqrt{6}}{5 \pi^{2}} \frac{\left(1+\sqrt{a_{22} / a_{11}}\right)^{5}}{\left|a_{12} / a_{11}+\sqrt{a_{22} / a_{11}}\right|^{5 / 2}} .
$$

To compare with the results of Ref. [38], the velocity $v$ can be expressed in the universal unit $\tilde{v}$ as

$$
\frac{v}{\tilde{v}}=\frac{5 \pi \hbar}{8 \sqrt{6} m a_{11}} \frac{\left|a_{12} / a_{11}+\sqrt{a_{22} / a_{11}}\right|^{3 / 2}}{\sqrt{a_{22} / a_{11}}\left(1+\sqrt{a_{22} / a_{11}}\right)^{3}} .
$$

We have also used a density functional derived from QMC calculations (QMC functional in the following), which is constructed by performing DMC calculations of a ${ }^{39} \mathrm{~K}$ mixture in the homogeneous phase [36]. The QMC functional $\mathcal{E}_{\text {int }}$ is obtained with the relation

$$
\mathcal{E}_{\text {int }}=\rho \frac{E}{N},
$$

where $E / N$ is the energy per particle of the extended system, calculated from QMC. QMC calculations were performed with the model potentials, which reproduce both the $s$-wave scattering lengths and effective ranges, which are known from the experiment [42]. In this way, the QMC functional correctly incorporates the two relevant scattering parameters of this mixture, i.e., the $s$-wave scattering lengths and the effective ranges.

\section{TIME-EVOLUTION EQUATIONS}

For a two-component system, our ansatz for the many-body wave function is

$$
\Psi\left(\mathbf{r}_{1}, \ldots, \mathbf{r}_{N} ; t\right)=\prod_{i=1}^{N_{1}} \psi_{1}\left(\mathbf{r}_{i}, t\right) \prod_{j=1}^{N_{2}} \psi_{2}\left(\mathbf{r}_{j}, t\right),
$$

where the number of particles of component 1 (2) is equal to $N_{1}\left(N_{2}\right)$. The equations of motion for the two components read

$$
i \hbar \frac{\partial \psi_{i}}{\partial t}=\mathcal{H}_{i} \psi_{i}=\left\{-\frac{\hbar^{2}}{2 m} \nabla^{2}+V_{i}\left(\rho_{1}, \rho_{2}\right)\right\} \psi_{i},
$$

for $i=1,2$, where $\rho_{i}=\left|\psi_{i}\right|^{2}$, and the potential $V_{i}$ is obtained from Eq. (1) [43].

$$
V_{i}=\frac{\partial \mathcal{E}\left[\rho_{1}, \rho_{2}\right]}{\partial \rho_{i}} .
$$

Explicitly, the coupled equations of motion for the two components of the condensate read

$$
\begin{aligned}
i \hbar \frac{\partial \psi_{1}}{\partial t}= & \left(-\frac{\hbar^{2}}{2 m} \nabla^{2}+\frac{4 \pi \hbar^{2} a_{11}}{m} \rho_{1}+\frac{4 \pi \hbar^{2} a_{12}}{m} \rho_{2}\right. \\
& \left.+\frac{128 \sqrt{\pi} \hbar^{2} a_{11}}{3 m}\left(a_{11} \rho_{1}+a_{22} \rho_{2}\right)^{3 / 2}\right) \psi_{1}, \\
i \hbar \frac{\partial \psi_{2}}{\partial t}= & \left(-\frac{\hbar^{2}}{2 m} \nabla^{2}+\frac{4 \pi \hbar^{2} a_{22}}{m} \rho_{2}+\frac{4 \pi \hbar^{2} a_{12}}{m} \rho_{1}\right. \\
& \left.+\frac{128 \sqrt{\pi} \hbar^{2} a_{22}}{3 m}\left(a_{11} \rho_{1}+a_{22} \rho_{2}\right)^{3 / 2}\right) \psi_{2} .
\end{aligned}
$$

When the ratio of densities is the optimal one, $\rho_{2} / \rho_{1}=$ $\sqrt{a_{11} / a_{22}}$, fixed by the condition of minimum energy per particle $[17,40,41]$, the energy functional for the total density $\rho=\rho_{1}+\rho_{2}$ reduces to

$$
\mathcal{E}_{\text {int }}=\alpha \rho^{2}+\beta \rho^{\gamma+1},
$$

where $\alpha, \beta$, and $\gamma$ are either the MF+LHY parameters from Eq. (6), or those that better fit the DMC equation of state [36]. In this case, the problem is thus effectively single component, meaning that the full many-body wave function (13) reduces to

$$
\Psi\left(\mathbf{r}_{1}, \boldsymbol{r}_{2}, \ldots, \mathbf{r}_{N} ; t\right)=\prod_{i=1}^{N} \psi\left(\mathbf{r}_{i} ; t\right),
$$

where $\psi$ is the solution of the following equation,

$$
i \hbar \frac{\partial \psi}{\partial t}=\mathcal{H} \psi=\left(\frac{-\hbar^{2}}{2 m} \nabla^{2}+V(\rho)\right) \psi,
$$

where

$$
V(\rho)=2 \alpha \rho+\beta(\gamma+1) \rho^{\gamma} .
$$

Equations (16), (17), and (20) are solved numerically by successively applying the time-evolution operator

$$
\psi(t+\Delta t)=e^{-i \mathcal{H} \Delta t} \psi(t),
$$

where a Trotter decomposition accurate to second order in the time step is implemented [44],

$$
e^{-i \mathcal{H} \Delta t / \hbar}=e^{-i \Delta t V\left(\mathbf{R}^{\prime}\right) / 2 \hbar} e^{-i \Delta t \hat{K} / \hbar} e^{-i \hbar \Delta t V(\mathbf{R}) / 2 \hbar}+\mathcal{O}\left(\Delta t^{3}\right),
$$

with $\hat{K}=-\hbar^{2} \nabla^{2} /(2 m)$. The kinetic energy propagator is evaluated in $k$ space by means of Fourier transforms.

Since an important parameter in our study is the deviation with respect to the optimal atom ratio $N_{2} / N_{1}=\sqrt{a_{11} / a_{22}}$, we define the atom ratio $x$ as

$$
x=\frac{N_{2}}{N_{1}} \sqrt{\frac{a_{22}}{a_{11}}},
$$

such that $x=1$ corresponds to the optimal mean-field composition. Note that one only needs to address the case $x \leqslant 1$ due to the symmetric role of atom numbers.

\section{REAL-TIME RELAXATION OF AN ISOLATED DROPLET}

We first focus our attention on the time evolution of a ${ }^{39} \mathrm{~K}-{ }^{39} \mathrm{~K}$ droplet as observed in the Florence 




FIG. 1. Time evolution of the total atom number $N$ in a stationary droplet. Lines are the results of two-component MF+LHY $+3 \mathrm{BL}$ calculations, and points are experimental data from Ref. [20]. The initial atom number is $N=2.5 \times 10^{5}$, with Gaussians of width $\sigma_{r}=3 \mu \mathrm{m}$ as density profiles. The values of $x=\left(N_{2} / N_{1}\right) \sqrt{a_{22} / a_{11}}$ imposed at $t=0$ are given in the top legend. The 3BL coefficients are shown in the panels in units of $\hbar /\left(m \xi^{2} \rho_{0}^{2}\right)$, with $\rho_{0}$ and $\xi$ being the equilibrium density and healing length given in Table I.

experiment (Fig. 2 in Ref. [20]). We performed twocomponent MF+LHY calculations with the goal to investigate the influence of the initial nonoptimal population ratio and $3 \mathrm{BL}$ on the real-time dynamics. To mimic the experimental setup, at $t=0$ we set the shape of a $N=2.5 \times 10^{5}$ atoms droplet as a Gaussian of width $\sigma_{r}=3 \mu \mathrm{m}$ centered in the origin and let the system evolve according to the extended Gross-Pitaevskii Eqs. (16) and (17). The scattering parameters $a_{i j}$ correspond to the magnetic field $B=56.54 \mathrm{G}$ (see Table I). We denote the $\left|F, m_{F}\right\rangle=|1,0\rangle$ state as component

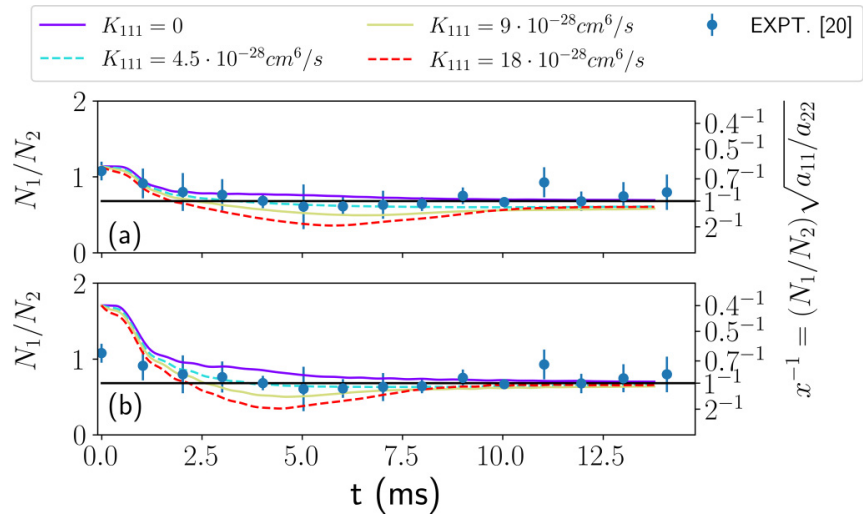

FIG. 2. Time evolution of $N_{1} / N_{2}$ for the same simulations as in Fig. 1. Starting atom number imbalances: (a), $x=0.6$; (b): $x=0.4$. Points are data from the Ref. [20]. The full black line is the corresponding $x=1$ atom ratio.

1 and the $\left|F, m_{F}\right\rangle=|1,-1\rangle$ state as component 2 . Within the two-component MF+LHY approach, we show in Fig. 1 the time evolution of the total atom number for different initial atom ratios within a broad range, namely, from $x=0.2$ to $x=1$. This was done changing the normalization of each component to the desired value at $t=0$, keeping fixed the total number of atoms, $N=2.5 \times 10^{5}$. It is worth noticing that the size of the whole simulation box is $60 \mu \mathrm{m}$, and we consider only the atoms inside a reduced box of $12 \mu \mathrm{m}$ to belong to the droplet and those outside of it as belonging to the surrounding cloud which arises as a consequence of the evaporation of the excess atoms in each species as time advances. We have verified that the total atom number within about $12 \mu \mathrm{m}$ box produces similar values, which are compatible with the experimental error bars shown in Fig. 1. We have included 3BL only in the time evolution of the $\psi_{1}$ component, whose $3 \mathrm{BL}$ coefficient is reported to be at least 100 times larger than the 3BL coefficients in the other channels $[20,45]$. This effect is included by introducing a term $-i \hbar K_{111}\left|\psi_{1}\right|^{4} / 2$ in Eq. (16). For each initial atom ratio, we have explored four different values of $K_{111}$, namely, $0,4.5 \times 10^{-28} \mathrm{~cm}^{6} / \mathrm{s}$, $9 \times 10^{-28} \mathrm{~cm}^{6} / \mathrm{s}$ and $18 \times 10^{-28} \mathrm{~cm}^{6} / \mathrm{s}$, respectively. These $K_{111}$ values span a broad range of possibilities, ranging from a fairly small to a rather large amount of 3BL. acting on the $\psi_{1}$ component.

Notice that there is a large experimental uncertainty affecting the actual value of $K_{111}: K_{111}$ values used in Figs. 1(b)-1(d) are compatible with the experimental error bar in the determination of the $3 \mathrm{BL}$ term for the ${ }^{39} \mathrm{~K}-{ }^{39} \mathrm{~K}$ mixture,

TABLE I. Scattering parameters, i.e., $s$-wave scattering lengths $a$, in units of Bohr radius $a_{0}$, as a function of the magnetic field $B$ [46]. $\xi$ and $\rho_{0}$ stand for healing length and equilibrium density of uniform liquid, respectively [see Eqs. (9) and (7)].

\begin{tabular}{llllll}
\hline \hline$B(\mathrm{G})$ & $a_{11}\left(a_{0}\right)$ & $a_{22}\left(a_{0}\right)$ & $a_{12}\left(a_{0}\right)$ & $\xi(\mu \mathrm{m})$ & $\rho_{0}\left(\mathrm{~cm}^{-3}\right)$ \\
\hline 56.23 & 63.648 & 34.587 & -53.435 & 0.496 & $5.773 \times 10^{15}$ \\
56.54 & 72.960 & 33.962 & -53.293 & 1.479 & $1.217 \times 10^{15}$ \\
56.55 & 73.3 & 33.9 & -53.3 & 1.527 & $1.164 \times 10^{15}$ \\
\hline \hline
\end{tabular}


which is $9 \times 10^{-28} \mathrm{~cm}^{6} / \mathrm{s}$, with an experimental uncertainty up to a factor of 2 [20]. For these $K_{111}$ values [panels (b) to (d) in Fig. 1], similar to those reported in Ref. [38], we observe that compared with the atom number imbalance $x, 3 \mathrm{BL}$ play a minor role in the equilibration process. The largest of the $K_{111}$ values Fig. 1(d) was used in the two-component calculations in Ref. [20] to explain the time evolution of the drop size observed in the experiment [20]. Looking at Fig. 1(d), it is clear that this $K_{111}$ value yields too strong 3BL.

The relative atom number obtained from our calculations is consistent with the experimental data for some $x$ and several values of $K_{111}$, as shown in Fig. 2, where we display the evolution of relative atom number, starting from $x=0.6$ [panel (a)] and $x=0.4$ [panel (b)] for four different $K_{111}$ values. From this analysis, we conclude that the observed atom loss can thus be mainly attributed to the droplet equilibration process in which the excess component is expelled until the optimal atom number ratio is eventually reached. A much lesser contribution to the equilibration process arises from $3 \mathrm{BL}$.

\section{DROPLET COLLISIONS}

We study the phase diagram characterizing the outcome of head-on binary collisions using the same description as in Ref. [38], i.e., in terms of $\left(v, N_{\text {coll }}\right)$, where $N_{\text {coll }}=N\left(t=t_{\text {coll }}\right)$ is the total atom number evaluated at the collision time $t_{\text {coll }}$, and $v$ is velocity of each droplet at the beginning of the simulation. The collision time $t_{\text {coll }}$ is estimated as $t_{\text {coll }}=d /(2 v)$, where $d$ is the initial distance between the two droplets. The initial wave function reads

$$
\psi(t=0)=\phi(x-d / 2, y, z) e^{+i k x}+\phi(x+d / 2, y, z) e^{-i k x},
$$

where $\phi$ and $k=m v / \hbar$ are the wave function and wave number of each droplet, respectively. Note that in all figures the total atom number $N$ and velocity $v$ is re-scaled according to Eqs. (10) and (11).

For all the simulations performed in this work, we have observed three possible collision outcomes: (i) For small velocities, merging of the droplets into a single one (coalescence appears after substantial deformation of the droplets); (ii) for higher velocities, separation, where the two droplets move away from one another after the collision; and (iii) evaporation, which occurs for very energetic collisions. Shattering is not observed because BEC drops must have a minimum size to be bound [17], and instead of a cloud of small droplets, the process continues until complete evaporation. These possibilities are illustrated in Fig. 3, where one may see the three outcomes: Merging [Fig. 3(a)], separation [Fig. 3(b)], and evaporation [Fig. 3(c)] The simulation corresponds to the two-component calculations described below.

In the experiments reported in Ref. [38], most of the merging events are found to lay below the dashed lines in Fig. 4 (the rising branch for low $N_{\text {coll }}$ and the decreasing one for higher $N_{\text {coll }}$ ), whereas most of the events leading to separation are found above these lines. Therefore in the following, when discussing the agreement between our theoretical predictions and experiments, we will use visual inspection to discriminate between good and bad agreement, considering a perfect agreement the case where the experimental dashed lines



FIG. 3. Three possible outcomes of a collision between quantum droplets simulated by two-component MF+LHY calculations; 3BL are not included. From left to right, the simulations correspond to $\tilde{v}=0.3,0.5$, and 1.1 for $\tilde{N}_{\text {coll }}=244$ in all three cases at the magnetic field $B=56.55 \mathrm{G}$. The panels show the integrated total atom density $\int d z \rho(\boldsymbol{r})$. The complete time evolution of the collisions is reported in Ref. [47].

separate the calculated merging points from those representing separation.

\section{A. Effective single-component calculations}

We have collected in Fig. 4 the collision outcomes obtained using the effective single-component MF+LHY and QMC functionals without including 3BL. The QMC functional, which depends on the magnetic field, is that corresponding to the magnetic field $B=56.23 \mathrm{G}$. For this $B$ value the difference between the MF+LHY and the QMC functionals we are using is the greatest [36]. Explicitly, this QMC functional is given by Eq. (18) with parameters $\alpha=-0.812 \hbar^{2} /\left(2 m a_{11}^{5}\right), \beta=$ $5.974 \hbar^{2} /\left(2 m a_{11}^{5-3 \gamma}\right)$, and $\gamma=1.276$, with $a_{11}=63.648 a_{0}$. 

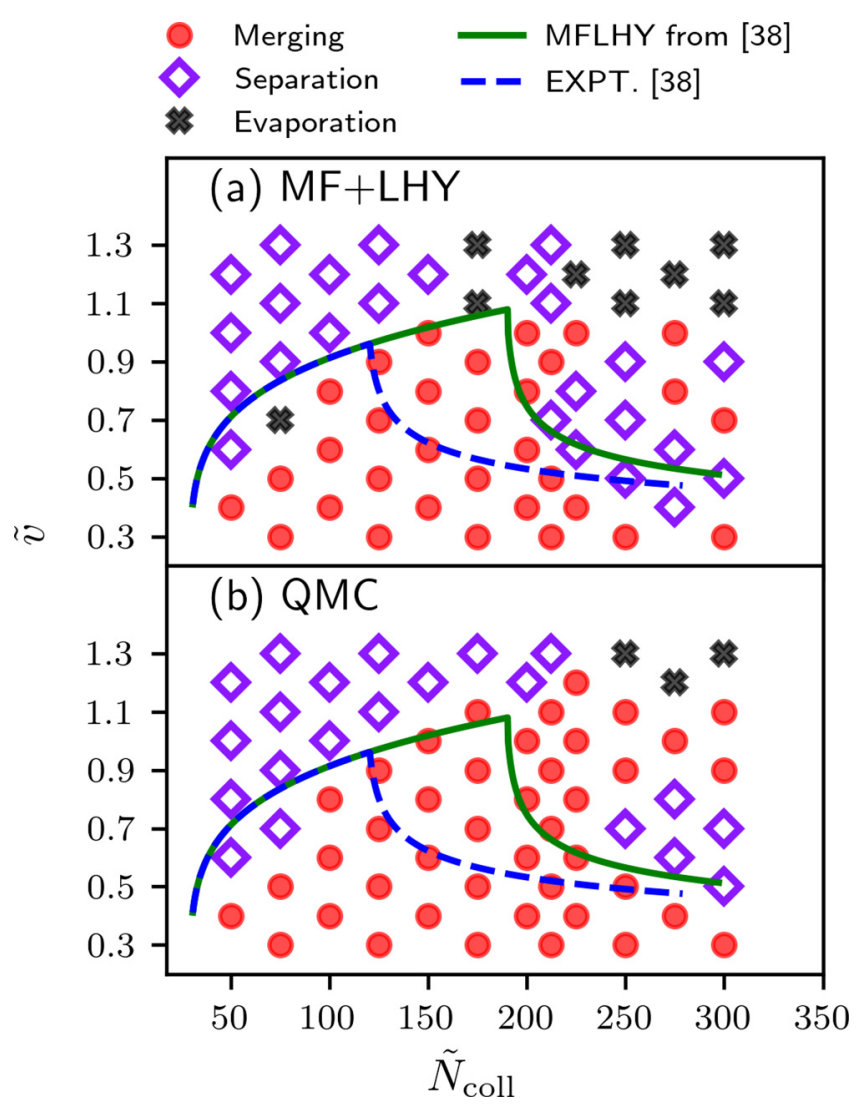

FIG. 4. Collision outcomes obtained using the effective singlecomponent MF+LHY (a) and QMC functional (b) with the scattering parameters corresponding to a magnetic field $B=56.23 \mathrm{G}$ (see Table I). In both cases, 3BL is not included. Atom number $N$ and velocity of each droplet at $t=0$ are re-scaled according to Eqs. (10) and (11), respectively. Points are collision outcomes, while dashed blue and full green lines are the empirical fits to the experimental and MF+LHY results without 3BL [38], respectively, for the velocity threshold above (below) which the droplets separate (merge).

For small $N$, the two functionals do not yield significantly different collision outcomes. There are some differences for big droplets, but not large enough to explain the deviation between theory and experiment observed in Ref. [38]. Merging is more likely to occur when using the QMC functional, which is somewhat expected since QMC functionals yield more binding [36] than the MF+LHY one, thus preventing the drops from separating.

From the results shown in Fig. 4 it appears that without including the 3BL, the effective single-component density functionals cannot account for the experimental observations since they predict droplets merging at much higher velocities than experimentally observed. This was one of the conclusions of the theoretical analysis in Ref. [38] within the MF+LHY approach, which we also find for QMC-based functionals.

\section{B. Two-component calculations}

We have performed collision simulations using the MF+LHY density functional in the two-component framework [Eqs. (16) and (17)]. Notice that this is not

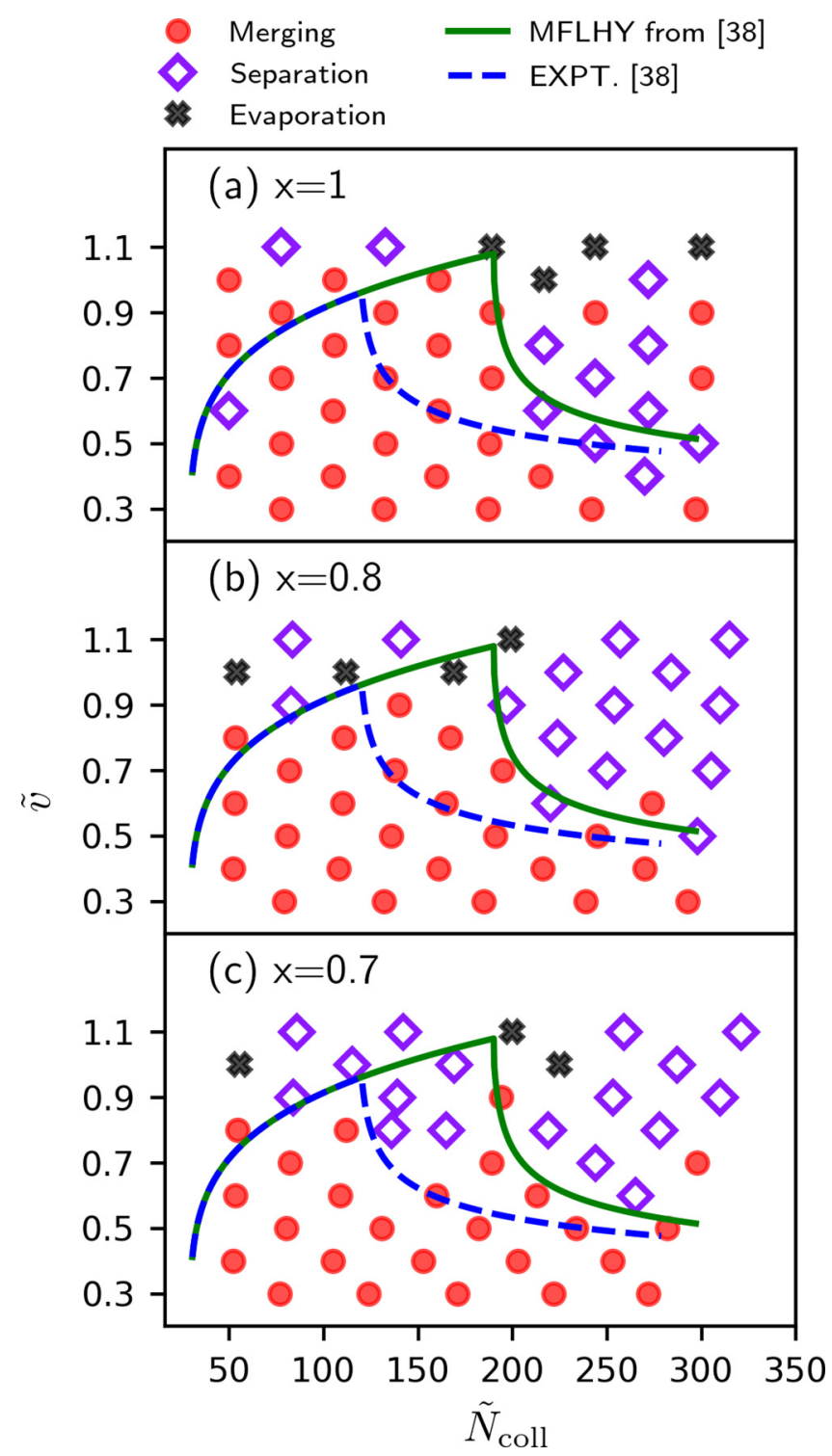

FIG. 5. Collision outcome as a function of droplet velocity $\tilde{v}$ and total atom number evaluated at the instant of collision $\tilde{N}_{\text {coll }}=\tilde{N}(t=$ $\left.t_{\text {coll }}\right)$ for the scattering parameters corresponding to $B=56.55 \mathrm{G}$. Calculations are performed using two-component MF+LHY theory without including 3BL. Parameter $x=\left(N_{2} / N_{1}\right) \sqrt{a_{22} / a_{11}}$ is the initial particle ratio at the beginning of collision. Points and lines have the same meaning as in Fig. 4.

possible with the present QMC functional, as it is written in terms of the total density alone. For each component, its wave function is evolved in time with the corresponding propagator [see Eqs. (14) and (22)].

\section{Neglecting $3 B L$}

We summarize in Fig. 5 the collision outcomes for several initial atom ratios $x$. The functional we use is that obtained from the scattering parameters $a_{i j}$ corresponding to the magnetic field $B=56.55 \mathrm{G}$ (see Table I), so our value is slightly above the range used in Ref. [38]. In all cases the initial droplet separation is $d \approx 3500 a_{11} \approx 9 \xi$, where $\xi$ is the corresponding healing length (see Table I). Since at $t=0$ 
the atom number ratio is not the optimal one, i.e., it does not correspond to the ground state of the extended GrossPitaevskii approach, the initial profile of each droplet has been prepared as follows. First, each droplet is equilibrated with optimal atom composition $x=1$ by means of imaginary-time propagation. Next, the real-time evolution is started and simultaneously the normalization of component 1 is changed to the desired value of $x$. When the collision is started with $x=1$ [Fig. 5(a)], the predictions within the two-component framework are in good agreement with the effective singlecomponent calculations of Ref. [38]. It can be seen that, as $x$ decreases [Figs. 5(a) and 5(b)], the merging region in the phase diagram shrinks and hence the $x \neq 1$ results are more similar to the experimental ones (we recall that $3 \mathrm{BL}$ are not included).

Note that there are small differences between the predictions obtained using effective single-component and twocomponent MF+LHY functionals assuming $x=1$. Since we are dealing with a finite system, the imposed requirement $\rho_{2} / \rho_{1}=\sqrt{a_{11} / a_{22}}$ satisfied in an effective single-component functional is not equally fulfilled in each spatial coordinate at the droplet surface in a two-component approach. Therefore, colliding drops display local deviations of the density ratio $\rho_{2} / \rho_{1}$, which eventually leads to the difference in collision outcomes.

Since $x \neq 1$ is not the equilibrium configuration, the atoms of the in-excess component are expelled out of the droplets as soon as time evolution starts. Therefore, the collision outcome depends on the initial distance $d$ between the two droplets (the larger the $d$, the larger the amount of atoms expelled from the droplets prior to colliding). Moreover, the evaporated atoms do not leave the collision region and may also influence the outcome. To highlight this important effect, we report in Fig. 6 the collision outcomes for nonequilibrated $(x=0.7)$ droplets and two different initial distances. Starting the collision at a large distance $(d=45 \xi)$, as in Fig. 6(b), the prediction for the critical velocity coincides with that obtained within the effective single-component framework. This is quite a natural result meaning that, by the time the two droplets meet, they have already reached a quasiequilibrium configuration where the ratio of atoms in each component corresponds to $x=1$.

\section{Effect of $3 B L$ and gas halo}

We have finally performed calculations including 3BL using the two-component MF+LHY density functional. Three-body recombination is assumed to be dominant in the $\left|F, m_{F}\right\rangle=|1,0\rangle$ channel, which we call state $1[20,45]$. Consequently, in our simulations, 3BL act only on $\psi_{1}$; Eq. (16).

We show in Fig. 7 the collision outcomes when the collision is started with the optimal atom number ratio $x=1$ [Fig. 7(a)] and with $x=0.8$ [Fig. 7(b)]. The initial density profiles and the distance between the two droplets is the same as in Fig. 5. The scattering parameters $a_{i j}$ correspond to the magnetic field $B=56.55 \mathrm{G}$. We choose a value of $K_{111}=2.73 \times 10^{-28} \mathrm{~cm}^{6} / \mathrm{s}=0.53 \hbar /\left(m \xi^{2} \rho_{0}^{2}\right)$, i.e., the same as in Ref. [38], where it has been observed that the effective single-component theory, supplemented with a 3BL term $-i \hbar K \rho^{2} / 2$, with $K=0.53 \hbar /\left(m \xi^{2} \rho_{0}^{2}\right)$ and $\rho$ being the total atom density, allowed to reproduce the experimental curve dividing merging from separation. Our calculations within



FIG. 6. Same as Fig. 5 for the nonoptimal atom number ratio $x=$ 0.7 and two different initial droplets distances. Left: Predictions of collision outcomes. Right: Initial droplets density profiles along the approaching direction, $\rho(x, 0,0 ; t=0)$. Full green and dashed blue lines have the same meaning as in Fig. 4. The top panel corresponds to $d=9 \xi$ and the bottom panel to $d=45 \xi$.

two-component formalism show that only when the initial atom number ratio is nonoptimal, namely, $x=0.8$, we observe separationlike outcomes of the collision and only in part of the phase diagram, for droplet velocities between $\tilde{v}=0.7$ and $\tilde{v}=1$. We have performed the calculations with the same initial conditions as in Fig. 7 but using the magnetic field $B=$ $56.3 G$, which is within the experimental window of magnetic fields used in Ref. [38], and $K_{111}=9.10^{-28} \mathrm{~cm}^{6} / \mathrm{s}$, and we see no significant differences with the results of Fig. 7.

To illustrate the effect of both the $3 \mathrm{BL}$ and nonoptimal atom ratios in more detail, we present in Fig. 8 four types of collisions obtained within the two-component MF+LHY functional. In all four cases, the integrated density $\rho_{i}(x, y)=$ $\int d z \rho_{i}(\boldsymbol{r})$ is shown, with component 1 (2) shown on the left (right).

In Figs. 8(a) and 8(b), the time evolution is shown without including three-body losses present in the system. The droplets are prepared with nonoptimal atom ratio $x=0.7$, as in Fig. 5. In both cases, separation is observed. For the smaller drops colliding at large velocity [Fig. 8(a)], drops evaporate on separation since they require a minimum critical atom number to be self-bound [17]. When atom numbers are slightly higher [Fig. 8(b)], the remaining part of the drops separates. In both cases, there is a sizable evaporation in component 1 when collision starts due to the initial population imbalance.

In Figs. 8(c) and 8(d) we show the influence of the initial atom ratio on the collision outcome including $3 \mathrm{BL}$ with $\tilde{K}_{111}=0.53$ for initial atom ratio equal to $x=1$ and $x=$ 0.8 , respectively. In this case, the interplay between threebody losses and atom number imbalance leads to different geometries during the collision, which eventually yield dif- 


\section{$\begin{array}{ll}\text { Merging } & \text { MFLHY from [38] } \\ \text { Separation } & -\end{array}$}

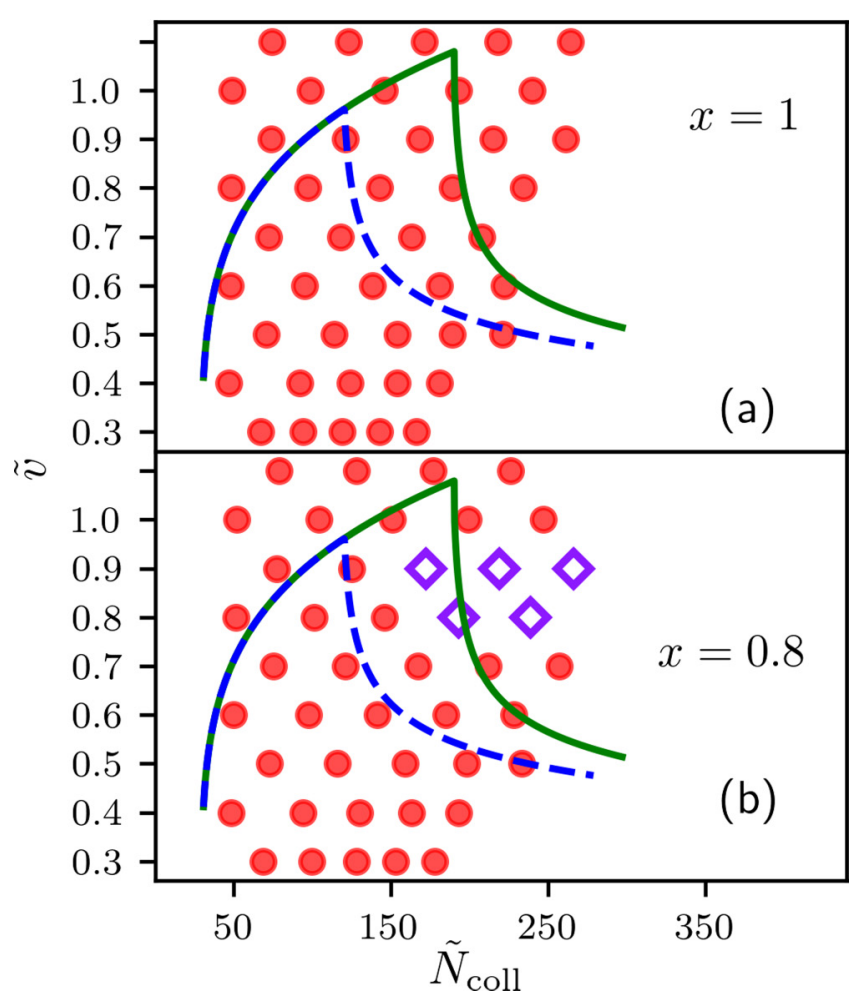

FIG. 7. Same as Fig. (5). Collision outcomes are predicted within the two-component MF+LHY theory with $3 \mathrm{BL}$ included in the $\left|F, m_{F}\right\rangle=|1,0\rangle$ component using $K_{111}=2.73 \times 10^{-28} \mathrm{~cm}^{6} / \mathrm{s}$, for two values of the initial atom number ratio is $x=\left(N_{2} / N_{1}\right) \sqrt{a_{22} / a_{11}}$.

ferent collision outcomes. When the initial atom ratio is $x=1$ [Fig. 8(c)], two protrusions are formed, reminiscent of collisions of He droplets $[8,11]$. The protrusions do not reach the size to be self-bound and eventually evaporate.

When the density at the droplets contact region increases, the density of component 1 diminishes due to $3 \mathrm{BL}$. This is followed by evaporation of component 2 due to the equilibration mechanism leading toward optimal particle number. This creates a halo around the droplets. Around $t=30 \mathrm{~ms}$, a shock wave is formed in component 2 as a result of the interference of outward expansion of the droplet with the surrounding halo. Eventually, the shock wave passes through the halo, and the drops remain at place. This result highlights that evaporated atoms that remain around the colliding droplets contribute to the merging process. Indeed, it has been experimentally found that coalescence of viscous droplets can be facilitated when the collision region contains atoms in the gas phase [2]. Notice that in order for the droplets to merge, the gas between them must be expelled, which costs some energy favoring the merging.

When the collision is started with $x=0.8$ [Fig. 8(d)], evaporation of component 1 in the early stage of collision takes place. This happens due to equilibration toward optimal particle composition, which would happen even in the absence of 3BL (see Fig. 1). Compared with the $x(t=0)=1$ case [Fig. 8(c)], a different droplet geometry and a thinner halo of species 2 appear at about $t=24 \mathrm{~ms}$. This is due to the fact that, when $x<1$, there is a time window in which only component 1 evaporates. The drops form a peanutlike configuration which has a much longer time life, also observed in Ref. [38], during which the large part of component 2 halo evaporates away. The peanut/cylinder eventually splits into two pieces. All of the separation collision outcomes observed in Fig. 7(b) are of the kind displayed in Fig. 8(d), indicating that this could be a common feature for collisions with initial population imbalance and 3BL present only in channel 111 .

We have also performed collisions using the effective single-component MF+LHY theory with the same value of three-body recombination as in Ref. [38], $\tilde{K}=0.53$, and we have found that the collision outcome (and thus the overall aspect of the $v-N$ phase diagram) is very sensitive to the initial preparation of the droplets, namely, the distance and the atom number at the start of the collision. In most cases our simulations predict separation of the droplet followed by evaporation, even for $(v, N)$ values for which merging is predicted in Ref. [38]. Thus, only a precise knowledge of how the droplets have been prepared (crucially, the initial droplet distance) would permit us to make a sensible comparison with the simulations of Ref. [38].

\section{SUMMARY AND CONCLUSIONS}

The recent study of head-on ${ }^{39} \mathrm{~K}-{ }^{39} \mathrm{~K}$ droplet collisions [38] offers a new avenue of research by extending the study of quantum droplet collisions-previously restricted to the case of helium droplets [32] to much lower density and temperature ranges of ultradilute cold Bose gas mixtures.

In the present work, we have taken this experiment as a case of study to theoretically analyze the influence of some elements not considered in the simulations carried out in that work. In particular, we have improved the density-functional approach by considering a functional based on QMC calculations that correctly incorporate the two relevant scattering parameters of the ${ }^{39} \mathrm{~K}-{ }^{39} \mathrm{~K}$ mixture, namely, the $s$-wave scattering length and the effective range, as well as the most general version of a two-component equal-masses Bose-Bose energy functional. This two-component functional allows to introduce the 3BL only in the most affected component of the mixture instead of in the total density of the system, which is usually unjustified. This is a crucial improvement over the effective single-component functionals like the QMC-based one, or the effective single-component MF+LHY functional which follows from the requirement that the two components of the mixture have the density ratio corresponding to the equilibrium one.

When 3BL are not considered, neither the QMC nor the effective single-component MF+LHY approaches agree with experiment. As already noted in the theoretical analysis of Ref. [38], the MF+LHY approach yields droplets merging at much higher velocities than observed experimentally. Moreover, the QMC-based and the single-component MF+LHY functionals meaningfully yield similar results. This is quite surprising since the QMC-based functional has a rather larger incompressibility and binding energy per atom than the single-component MF+LHY functional [36], which was the reason to use it in this study. 


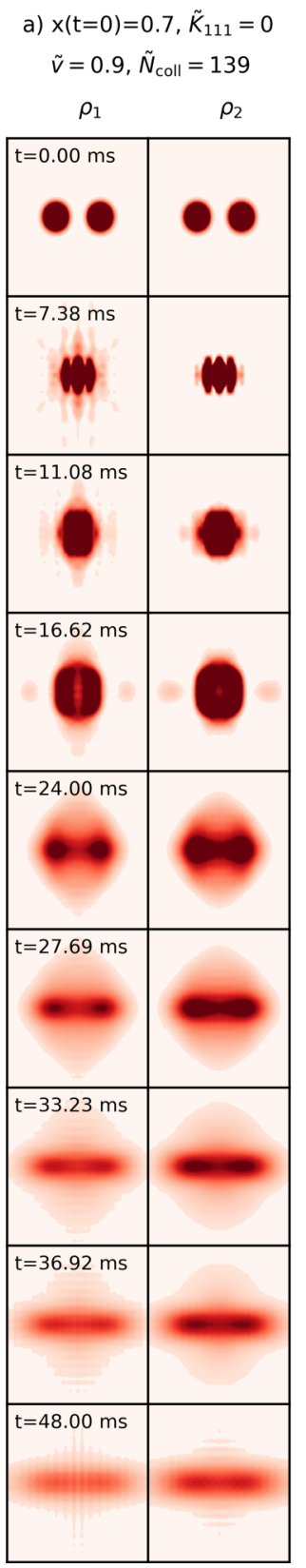

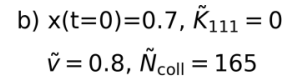
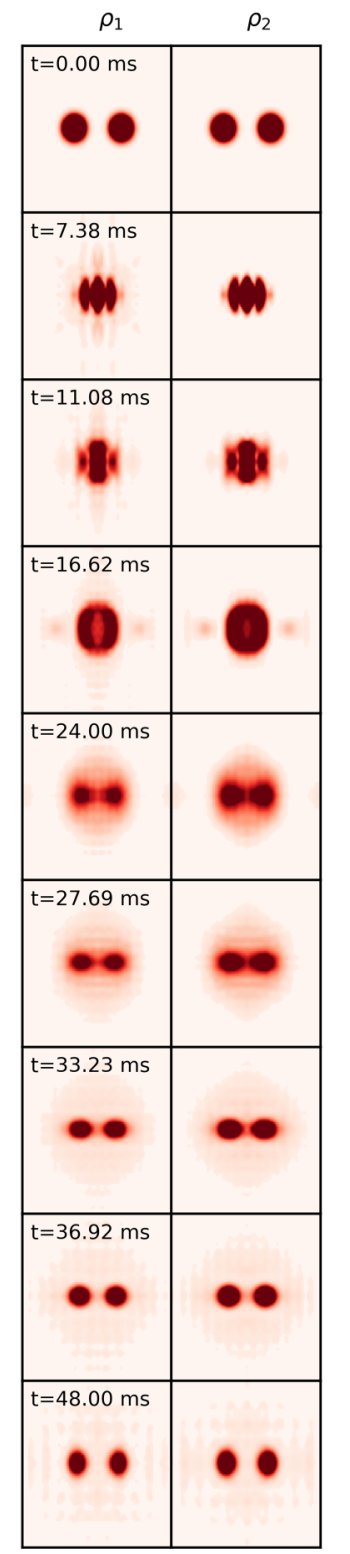



c) $x(t=0)=1, \tilde{K}_{111}=0.53$

$\tilde{v}=0.9, \tilde{N}_{\text {coll }}=215$




$\rho_{1}$

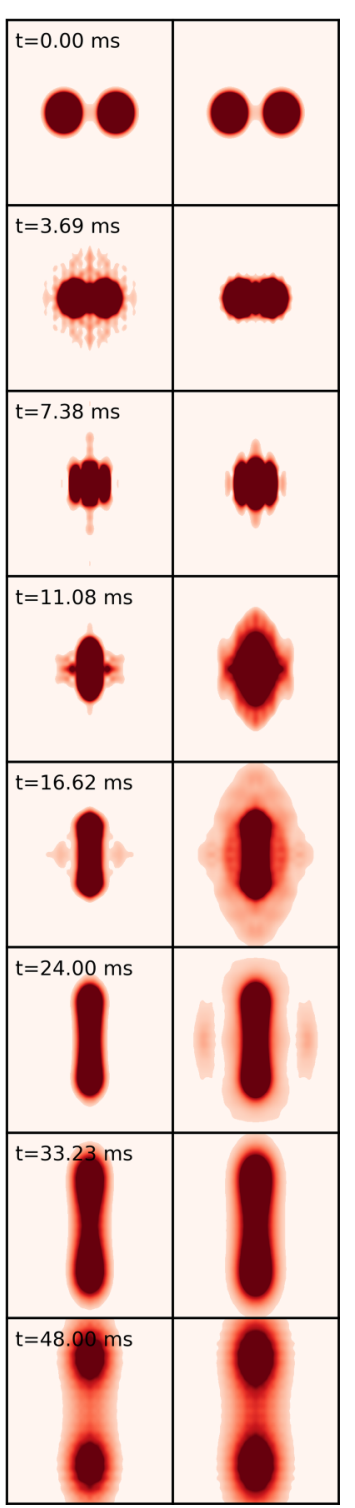

FIG. 8. Images of the time evolution of $\rho_{i}=\int d z \rho_{i}(\boldsymbol{r})$, for components $i=1$ and 2, obtained within the two-component MF+LHY functional. The complete time-dependent evolution of the collisions is reported in Ref. [47].

It might well be that using a $K_{111}$ coefficient similar or compatible with a given experiment, one finds a poor agreement with the experimental results. In this case, a natural possibility is that droplets are not fully equilibrated when the collision is considered to have started. We have thus completed our analysis, assuming that the droplets are not in equilibrium at the start of the collision, including in some cases 3BL but always only in the most affected component.

If the droplets are not fully equilibrated when they enter the collision region, the outcome sensibly depends on three key parameters: (i) Initial droplete distance, (ii) initial atom ratio, and (iii) the value of the $3 \mathrm{BL}$ coefficient. Furthermore, when we use nonoptimal atom ratios, we observed an important additional effect not previously considered, namely, the halo of the expelled gaseous particles of the in-excess species that envelop the droplets and increase the tendency to merge. This effect has also been found in viscous droplet collisions [2]. Remarkably, the phase diagram changes even without the introduction of 3BL, as collisions between nonequilibrated droplets are shown to behave differently from equilibrated ones in terms of the optimal atom number ratio. Obviously, the introduction of considerations of 3BL and/or nonequilibrium configuration at $t=0$ in the description of the collision process has a dramatic impact on the outcome of the simulations and adds elements of difficult control when comparing with experiment. We have focused here on zero temperature description, but it is plausible that thermally excited droplets [48] could produce similar shifts in the critical velocity. 
Finally, we want to emphasize that while 3BL and nonequilibrium effects reduce the number of atoms and the kinetic energy in the colliding droplets, both effects are by no means equivalent. The term added to the functional to introduce $3 \mathrm{BL}$ takes atoms and energy out of the computational box, while the atoms of the in-excess component remain in the collision region and their presence may affect the collision outcome.

\section{ACKNOWLEDGMENTS}

This work has been supported by the Ministerio de Economia, Industria y Competitividad (MINECO, Spain) under Grants No. FIS2017-84114-C2-1-P and No. PID2020114626GB-I00 and by the EC Research Innovation Action under the H2020 Programme, Project No. HPC-EUROPA3 (INFRAIA-2016-1-730897). V.C. gratefully acknowledges the computer resources and technical support provided by Barcelona Supercomputing Center. We also acknowledge financial support from Secretaria d'Universitats i Recerca del Departament d'Empresa i Coneixement de la Generalitat de Catalunya, cofunded by the European Union Regional Development Fund within the ERDF Operational Program of Catalunya (Project QuantumCat, Ref. 001-P-001644).
[1] N. Ashgriz and J. Y. Poo, Coalescence and separation in binary collisions of liquid drops, J. Fluid Mech. 221, 183 (1990).

[2] J. Qian and C. K. Law, Regimes of coalescence and separation in droplet collision, J. Fluid Mech. 331, 59 (1997).

[3] K.-L. Pan, P.-C. Chou, and Y.-J. Tseng, Binary droplet collision at high Weber number, Phys. Rev. E 80, 036301 (2009).

[4] V. B. Varma, A. Ray, Z. M. Wang, Z. P. Wang, and R. V. Ramanujan, Droplet merging on a lab-on-a-chip platform by uniform magnetic fields, Sci. Rep. 6, 37671 (2016).

[5] W. J. Swiatecki, The dynamics of the fusion of two nuclei, Nucl. Phys. A 376, 275 (1982).

[6] M. Guilleumas, M. Pi, M. Barranco, and E. Suraud, 3 he-3 he drop collisions in the vlasov dynamics, Z. Phys. D 34, 35 (1995).

[7] J. P. Toennies and A. F. Vilesov, Superfluid helium droplets: A uniquely cold nanomatrix for molecules and molecular complexes, Angew. Chem., Int. Ed. 43, 2622 (2004).

[8] C. L. Vicente, C. Kim, H. J. Maris, and G. M. Seidel, Coalescence of levitated he ii drops, J. Low Temp. Phys. 121, 627 (2000).

[9] H. J. Maris, Analysis of the early stage of coalescence of helium drops, Phys. Rev. E 67, 066309 (2003).

[10] R. Ishiguro, F. Graner, E. Rolley, and S. Balibar, Coalescence of Crystalline Drops, Phys. Rev. Lett. 93, 235301 (2004).

[11] J. M. Escartín, F. Ancilotto, M. Barranco, and M. Pi, Vorticity and quantum turbulence in the merging of superfluid helium nanodroplets, Phys. Rev. B 99, 140505(R) (2019).

[12] S. Nazarenko, Wave turbulence, Contemp. Phys. 56, 359 (2015).

[13] M. Tsubota and W. P. Halperin, Bose-Einstein Condensation and Superfluidity, Vol. XVI (Progress in Low Temperature Physics, Elsevier, Amsterdam, 2009).

[14] B. Sun and M. S. Pindzola, Slow collisions of Bose-Einstein condensates in both ground and excited states, J. Phys. B: At., Mol. Opt. Phys. 41, 155302 (2008).

[15] G. E. Astrakharchik and B. A. Malomed, Dynamics of onedimensional quantum droplets, Phys. Rev. A 98, 013631 (2018).

[16] J. Lao, Z. Zhou, X. Zhang, F. Ye, and H. Zhong, Oscillatory stability of quantum droplets in $\mathcal{P} \mathcal{T}$-symmetric optical lattice, Commun. Theor. Phys. 73, 065103 (2021).

[17] D. S. Petrov, Quantum Mechanical Stabilization of a Collapsing Bose-Bose Mixture, Phys. Rev. Lett. 115, 155302 (2015).

[18] C. R. Cabrera, L. Tanzi, J. Sanz, B. Naylor, P. Thomas, P. Cheiney, and L. Tarruell, Quantum liquid droplets in a mixture of Bose-Einstein condensates, Science 359, 301 (2018).

[19] P. Cheiney, C. R. Cabrera, J. Sanz, B. Naylor, L. Tanzi, and L. Tarruell, Bright Soliton to Quantum Droplet Transition in a Mixture of Bose-Einstein Condensates, Phys. Rev. Lett. 120, 135301 (2018).

[20] G. Semeghini, G. Ferioli, L. Masi, C. Mazzinghi, L. Wolswijk, F. Minardi, M. Modugno, G. Modugno, M. Inguscio, and M. Fattori, Self-Bound Quantum Droplets of Atomic Mixtures in Free Space, Phys. Rev. Lett. 120, 235301 (2018).

[21] C. D’Errico, A. Burchianti, M. Prevedelli, L. Salasnich, F. Ancilotto, M. Modugno, F. Minardi, and C. Fort, Observation of quantum droplets in a heteronuclear bosonic mixture, Phys. Rev. Research 1, 033155 (2019).

[22] T. D. Lee and C. N. Yang, Many-body problem in quantum mechanics and quantum statistical mechanics, Phys. Rev. 105, 1119 (1957).

[23] K. Huang and C. N. Yang, Quantum-mechanical many-body problem with hard-sphere interaction, Phys. Rev. 105, 767 (1957).

[24] D. M. Larsen, Binary mixtures of dilute Bose gases with repulsive interactions at low temperature, Ann. Phys. 24, 89 (1963).

[25] F. Minardi, F. Ancilotto, A. Burchianti, C. D'Errico, C. Fort, and M. Modugno, Effective expression of the Lee-Huang-Yang energy functional for heteronuclear mixtures, Phys. Rev. A 100, 063636 (2019).

[26] P. Naidon and D. S. Petrov, Mixed Bubbles in Bose-Bose Mixtures, Phys. Rev. Lett. 126, 115301 (2021).

[27] D. S. Petrov and G. E. Astrakharchik, Ultradilute LowDimensional Liquids, Phys. Rev. Lett. 117, 100401 (2016).

[28] L. Parisi, G. E. Astrakharchik, and S. Giorgini, Liquid State of One-Dimensional Bose Mixtures: A Quantum Monte Carlo Study, Phys. Rev. Lett. 122, 105302 (2019).

[29] L. Parisi and S. Giorgini, Quantum droplets in one-dimensional Bose mixtures: A quantum Monte Carlo study, Phys. Rev. A 102, 023318 (2020).

[30] F. Böttcher, M. Wenzel, J.-N. Schmidt, M. Guo, T. Langen, I. Ferrier-Barbut, T. Pfau, R. Bombín, J. Sánchez-Baena, J. Boronat, and F. Mazzanti, Dilute dipolar quantum droplets beyond the extended Gross-Pitaevskii equation, Phys. Rev. Research 1, 033088 (2019).

[31] F. Böttcher, J.-N. Schmidt, J. Hertkorn, K. S. H. Ng, S. D. Graham, M. Guo, T. Langen, and T. Pfau, New states of matter with fine-tuned interactions: quantum droplets 
and dipolar supersolids, Rep. Prog. Phys. 84, 012403 (2021).

[32] M. Barranco, R. Guardiola, S. Hernández, R. Mayol, J. Navarro, and M. Pi, Helium nanodroplets: An overview, J. Low Temp. Phys 142, 1 (2006).

[33] H. Hu and X.-J. Liu, Consistent Theory of Self-Bound Quantum Droplets with Bosonic Pairing, Phys. Rev. Lett. 125, 195302 (2020).

[34] S. Giorgini, J. Boronat, and J. Casulleras, Ground state of a homogeneous Bose gas: A diffusion Monte Carlo calculation, Phys. Rev. A 60, 5129 (1999).

[35] M. Rossi and L. Salasnich, Path-integral ground state and superfluid hydrodynamics of a bosonic gas of hard spheres, Phys. Rev. A 88, 053617 (2013).

[36] V. Cikojević, L. V. Markić, and J. Boronat, Finite-range effects in ultradilute quantum drops, New J. Phys. 22, 053045 (2020).

[37] V. Cikojević, L. Vranješ Markić, G. E. Astrakharchik, and J. Boronat, Universality in ultradilute liquid Bose-Bose mixtures, Phys. Rev. A 99, 023618 (2019).

[38] G. Ferioli, G. Semeghini, L. Masi, G. Giusti, G. Modugno, M. Inguscio, A. Gallemí, A. Recati, and M. Fattori, Collisions of Self-Bound Quantum Droplets, Phys. Rev. Lett. 122, 090401 (2019).

[39] A. Frohn and N. Roth, Dynamics of Droplets (Springer Science $\&$ Business Media, Springer, Berlin, 2000).

[40] C. Staudinger, F. Mazzanti, and R. E. Zillich, Self-bound Bose mixtures, Phys. Rev. A 98, 023633 (2018).
[41] F. Ancilotto, M. Barranco, M. Guilleumas, and M. Pi, Self-bound ultradilute Bose mixtures within local density approximation, Phys. Rev. A 98, 053623 (2018).

[42] L. Tanzi, C. R. Cabrera, J. Sanz, P. Cheiney, M. Tomza, and L. Tarruell, Feshbach resonances in potassium Bose-Bose mixtures, Phys. Rev. A 98, 062712 (2018).

[43] M. Barranco, F. Coppens, N. Halberstadt, A. Hernando, A. Leal, D. Mateo, R. Mayol, and M. Pi, Zero temperature DFT and TDDFT for $4 \mathrm{He}$ : A short guide for practitioners, https://github. com/bcntls2016/DFT-Guide/blob/master/dft-guide.pdf (2017).

[44] S. A. Chin, S. Janecek, and E. Krotscheck, Any order imaginary time propagation method for solving the schrödinger equation, Chem. Phys. Lett. 470, 342 (2009).

[45] G. Ferioli, G. Semeghini, S. Terradas-Briansó, L. Masi, M. Fattori, and M. Modugno, Dynamical formation of quantum droplets in a ${ }^{39} \mathrm{~K}$ mixture, Phys. Rev. Research 2, 013269 (2020).

[46] S. Roy, M. Landini, A. Trenkwalder, G. Semeghini, G. Spagnolli, A. Simoni, M. Fattori, M. Inguscio, and G. Modugno, Test of the Universality of the Three-Body Efimov Parameter at Narrow Feshbach Resonances, Phys. Rev. Lett 111, 053202 (2013).

[47] See Supplemental Material at http://link.aps.org/supplemental/ 10.1103/PhysRevResearch.3.043139 for videos of the time evolution of selected collisions.

[48] J. Wang, H. Hu, and X.-J. Liu, Thermal destabilization of selfbound ultradilute quantum droplets, New J. Phys. 22, 103044 (2020). 